\title{
Analysis of Phase Velocity of Love Waves in Rigid and Soft Mountain Surfaces: Exponential Law Model
}

\author{
Uma Bharti, ${ }^{1}$ Pramod Kumar Vaishnav ${ }^{1},{ }^{1}$ S.M. Abo-Dahab, ${ }^{2,3}$ Jamel Bouslimi, ${ }^{4}$ \\ and K.H. Mahmoud ${ }^{5}$ \\ ${ }^{1}$ School of Mathematics, Thapar Institute of Engineering and Technology, Patiala-147001, India \\ ${ }^{2}$ Department of Mathematics, Faculty of Science, South Valley University, Qena-83523, Egypt \\ ${ }^{3}$ Department of Computer Science, Faculty of Computers and Information, Luxor University, Armant, Egypt \\ ${ }^{4}$ Department of Physics, College of Science, Taif University, Taif, Saudi Arabia \\ ${ }^{5}$ Department of Physics, College of Khurma University College, Taif University, Taif, Saudi Arabia \\ Correspondence should be addressed to Pramod Kumar Vaishnav; pvaishnav.ism@gmail.com
}

Received 16 March 2021; Accepted 4 May 2021; Published 19 May 2021

Academic Editor: Ahmed Mostafa Khalil

Copyright ( $\odot 2021$ Uma Bharti et al. This is an open access article distributed under the Creative Commons Attribution License, which permits unrestricted use, distribution, and reproduction in any medium, provided the original work is properly cited.

\begin{abstract}
Irregularity may occur on the earth's surface in the form of mountains due to the imperfection of the earth's crust. To explore the influence of horizontally polarized shear waves on mountains, we considered the fluid-saturated porous medium (superficial layer) over an orthotropic semi-infinite medium with rigid (Model-I) and soft (Model-II) mountain surfaces for wave propagation. The mountain surface is defined mathematically as a periodic function of the time domain. The physical interpretation of materials' structure has been explained in rectangular Cartesian coordinate system originated at the contact interface of layer and half-space. The displacement of the mountains has been derived by solving energy equations analytically. The influence of rigid and soft mountain surfaces on the phase velocity of shear waves has been demonstrated graphically (we used MATLAB software for graphical representations).
\end{abstract}

\section{Introduction}

Imperfection of the earth's surface play an important role to maintain the environmental requirements, which lead us to investigate the impact of Love wave propagation under mountain surfaces. From the literature, it has been noticed that mountains have rigid and soft surfaces. Within finite dimension and boundaries, the Earth is of spherical shape which has irregular surfaces, composite materials, and high initial stresses. Love waves, named after Augustus Edward Hough Love, are horizontally polarized surface waves which propagate in the presence of superficial layer of finite thickness overlying half-space. Earth's surface may shift horizontally due to Love waves' propagation which is highly responsible for the damaging of the buildings during an earthquake. These waves are observed only when $c_{1}<c_{2}\left(c_{1}\right.$ and $c_{2}$ are phase velocities of layer and half-space, respectively). To understand the Earth's composition, researchers have used different Earth models under different physical conditions, e.g., inhomogeneity, magnetoelasticity, anisotropy, porosity, reinforcement, and irregularity. Seismic wave propagation is documented by Love [1], Biot [2], Ewing et al. [3], and Gubbins [4].

The initial stress is the hydrostatic stress that is generated inside the Earth due to high temperature, gravitating pull, pressure, etc. To expose the effect of initial stress on the phase velocity, the earth layers can be considered under high initial stress for the propagation of Love waves. The elastic waves produced during the time of earthquake show a remarkable effect due to presence of initial stress in a fluid saturated porous layer. Many researchers contributed a great amount of theoretical work on elastic wave propagation in Earth medium under the effect of initial stresses. The influence of initial stress on elastic wave propagation under the plain Earth surfaces has been investigated by Abd-Alla et al. [5]. The authors concluded that the presence of initial stress 
in the medium may affect the propagation speed scientifically. Gupta et al. [6] demonstrated the effect of initial stress on propagation of Love waves in an anisotropic porous layer under the vacuum surface of the Earth. Ahmed and AbdDahab [7] examined the effect of initial stress on the propagation of Love waves in an orthotropic granular layer lying over a semi-infinite granular medium. Gupta et al. [8] explained the influence of rigid boundary and initial stress on the propagation of Love wave. Ogden and Singh [9] discussed the propagation of waves in an incompressible transversely isotropic elastic solid with initial stress. AbdAlla et al. [10] investigated the propagation of Love waves in a nonhomogeneous orthotropic magnetoelastic layer under initial stress. Gupta et al. [11] studied the propagation of Love waves in a nonhomogeneous substratum over initially stressed heterogeneous half-space. Ogden and Singh [12] investigated the effect of rotation and initial stress on the propagation of waves in a transversely isotropic elastic solid. Kakar and Kakar [13] demonstrated the effect of gravity, initial stress, and electric and magnetic field on the propagation of S-wave in an anisotropic porous half-space. Pal and Ghorai [14] discussed the Love wave propagation in sandy layer under initial stress above anisotropic porous half-space under gravity. Sham [15] investigated propagation of wave under the initial stress at the boundary between a layer and a half-space. Nam et al. [16] showed the impact of initial stress in the layered half-space on the propagation of surface waves. Ejaz and Shams [17] analyzed the initial stress in compressible elastic materials on the wave propagation. Kundu et al. [18] generalized the effect of initial stress on the propagation and attenuation characteristics of Rayleigh waves. Tochhawng and Singh [19] observed the effect of initial stresses on the elastic waves in transversely isotropic thermoelastic materials. The above said papers have discussed the effect of initial stress on wave propagation under the plain Earth surfaces; however, it is realized that the initial stress may affect the phase velocity of the Love wave under irregular rigid and soft mountain surfaces. Keeping this in mind, we considered the periodic irregular surface of superficial layer for Love wave propagation in composite material structure. The propagation of elastic wave in fluid saturated porous medium, reinforced medium, and orthotropic medium has been discussed by several researchers. Ke et al. [20] illustrated the wave propagation with the properties varying exponentially in an inhomogeneous fluid saturated porous layered half-space, Ghorai et al. [21] examined the effect of gravity on the Love waves propagation in a fluid-saturated porous layer under a rigid boundary and lying over an elastic half-space, Chen et al. [22] theoretically analyzed the wave propagation an unsaturated porous medium, Kumar and Rajeev [23] studied analysis of wave motion at the boundary surface of orthotropic thermoelastic material with voids and isotropic elastic half-space, Gupta et al. [24] identified the possibility of Love wave propagation in a porous layer under the effect of linearly varying directional rigidities, Vaishnav et al. [25] studied about the rectangular irregularity in propagation of Love-type wave in porous medium over an orthotropic semi-infinite medium, Manna et al. [26] clearly defined the effect of reinforcement and inhomogeneity on the propagation of Love wave, Saha et al. [27] exposed the torsional surface waves in an initially stressed porous layer sandwiched between two nonhomogeneous half-spaces with irregularity, and Kumhar et al. [28] proposed an Earth model for Love waves in fluid saturated porous viscoelastic medium resting over an exponentially graded inhomogeneous half-space influenced by gravity. Li et al. [29] and Ju et al. [30] discussed the cognition on earthquake and vibration control of a mobile flexible manipulator via synchronization optimization of observation and feedback. The Earth's surface is irregular in nature which really affects the propagation of seismic waves by all means. So, several studies on elastic wave propagation under the effect of irregular boundaries have been documented in the literature.

We have considered the fluid saturated porous medium over an heterogeneous orthotropic half-space in Model-I and Model-II to explore the influence of rigid and soft mountain surfaces on the phase velocity of Love wave. Love wave dispersion relation has been obtained for both models, for which the elastic coefficients of the orthotropic medium obey the exponential law model $\left(E_{x} e^{\bar{\alpha} z}, E_{y} e^{\bar{\alpha} z}, E_{z} e^{\bar{\alpha} z}, G_{x y} e^{\bar{\alpha} z}, G_{y z} e^{\bar{\alpha} z}, G_{z x} e^{\bar{\alpha} z}\right.$, and $\left.\rho^{m_{2}} e^{\bar{\alpha} z}\right)$. The dynamics of fluid saturated porous medium and exponential varying orthotropic medium was studied by Kundu et al. [31], Wang et al. [32], and Vaishnav et al. [33]. The effects of initial stress, heterogeneity parameters, and irregularity have been demonstrated graphically for both cases using MATLAB software. The obtained generalized dispersion relation is identical to the classical dispersion relation of Love wave. Hence, it is imperative to take periodic irregularity into consideration for the evaluation of Love waves' propagation under the mountain surfaces.

\section{Formulation of the Problem}

In this paper, we consider the theoretical analysis of the phase velocity of the Love waves in rigid and soft mountain surfaces. The propagation medium for the wave is assumed as fluid saturated porous layer of finite thickness $H$ lying over an orthotropic semi-infinite medium, as illustrated in Figure 1. The origin of the rectangular Cartesian coordinate system is taken at the contact interface of layer and halfspace and the direction of wave propagation is taken along $x$-axis and antiplane shear motion is along the $y$-direction, and $z$-axis is taken vertically downwards to the direction of wave propagation. The mountain surfaces may define periodic irregularity on the surface of the superficial layer as provided by Singh [34]. The periodic irregularity (mountain surface) is considered for both models:

$$
\lambda_{j}(x)=\sum_{n=1}^{\infty}\left(\lambda_{n}^{j} e^{\mathrm{inpx}}+\lambda_{-n}^{j} e^{-\mathrm{inpx}}\right), j=1,2,
$$

where $\lambda_{n}^{j}$ and $\lambda_{-n}^{j}$ are Euler coefficients, $p$ is the wavenumber, $n$ is the order of series, and $i=\sqrt{-1}$. The amplitude of irregular boundaries is considered very small compared with the wavelength $2 \pi / p$. 


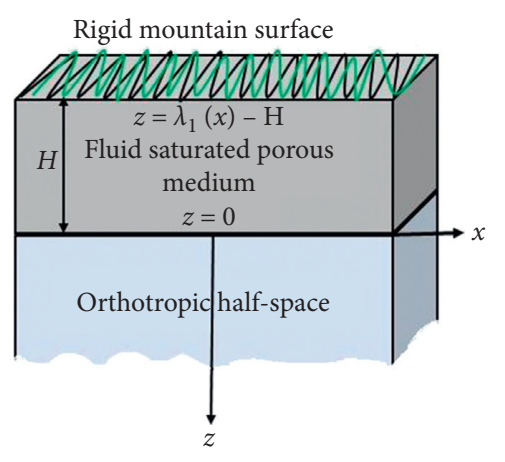

(a)

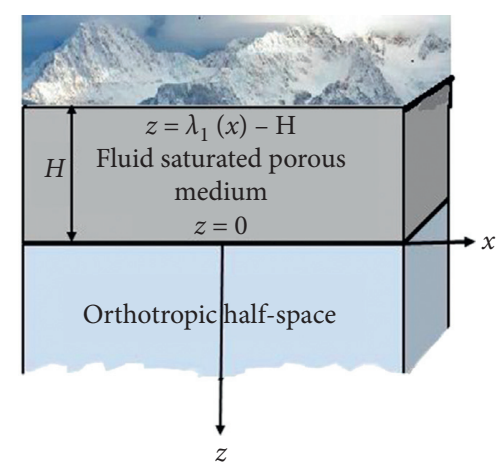

(b)

Figure 1: Model-I: medium of the propagation under rigid mountain surface.

\section{Phase Velocity Equations for Initially Stressed Fluid Saturated Porous Medium}

The stress-strain relation for the solid and fluid saturated porous materials is

$$
\left.\begin{array}{l}
S_{x x}^{M_{1}}=P\left(e_{x x}+e_{y y}+e_{z z}\right)+\lambda^{M_{1}}\left(e_{11}+e_{22}\right)-2 \mathrm{Ne}_{22}+\mu^{M_{1}} e_{33}+\mathrm{CE}, \\
S_{y y}^{M_{1}}=\lambda^{M_{1}}\left(e_{11}+e_{22}\right)-2 \mathrm{Ne}_{11}+\mu^{M_{1}} e_{33}+\mathrm{CE}, \\
S_{z z}^{M_{1}}=\mu^{M_{1}}\left(e_{11}+e_{22}\right)+T e_{33}+\mathrm{CE}, \\
S_{x y}^{M_{1}}=2 \mathrm{Ne}_{x y}, \\
S_{y z}^{M_{1}}=2 \mathrm{Le}_{y z}, \\
S_{x z}^{M_{1}}=2 \mathrm{Le}_{z x},
\end{array}\right\}
$$$$
S_{y y}^{M_{1}}=\lambda^{M_{1}}\left(e_{11}+e_{22}\right)-2 \mathrm{Ne}_{11}+\mu^{M_{1}} e_{33}+\mathrm{CE},
$$

where $e_{i j}=(1 / 2)\left(\left(\partial u_{i} / \partial x_{j}\right)+\left(\partial u_{j} / \partial x_{i}\right)\right)$ are strain components, $E=\sum_{i=1}^{3}\left(\mathrm{~d} U_{i} / \mathrm{d} x_{i}\right), i, j=1,2,3, S_{i j}^{M_{1}}$ are stress components, $\lambda^{M_{1}}, \mu^{M_{1}}$, and $T$ are elastic coefficients, $N$ and $L$ are rigidities of anisotropy along $x$ - and $z$-directions, respectively, and $P$ represents the initial stress due to high temperature of the medium. The positive coefficient $C$ denoted the measure of coupling between the change of volume of solid and fluid.

3.1. Energy Equations. The energy equations for the upper layer in the absence of body forces and viscosity are given by Biot [4] as

$\% \frac{\partial S_{11}^{M_{1}}}{\partial x}+\frac{\partial S_{12}^{M_{1}}}{\partial y}+\frac{\partial S_{13}^{M_{1}}}{\partial z}-P\left(\frac{\partial w_{z}}{\partial y}-\frac{\partial w_{y}}{\partial z}\right)=\frac{\partial^{2}}{\partial t^{2}}\left(\rho_{11} U_{1}+\rho_{12} U_{x}\right)$

$$
\frac{\partial S_{21}^{M_{1}}}{\partial x}+\frac{\partial S_{22}^{M_{1}}}{\partial y}+\frac{\partial S_{23}^{M_{1}}}{\partial z}-P\left(\frac{\partial w_{z}}{\partial x}\right)=\frac{\partial^{2}}{\partial t^{2}}\left(\rho_{11} V_{1}+\rho_{12} V_{y}\right)
$$

$$
\begin{aligned}
\frac{\partial S_{31}^{M_{1}}}{\partial x}+\frac{\partial S_{32}^{M_{1}}}{\partial y}+\frac{\partial S_{33}^{M_{1}}}{\partial z}-P\left(\frac{\partial w_{y}}{\partial x}\right)=\frac{\partial^{2}}{\partial t^{2}}\left(\rho_{11} W_{1}+\rho_{12} W_{z}\right) \\
\frac{\partial S}{\partial x}=\frac{\partial^{2}}{\partial t^{2}}\left(\rho_{11} U_{1}+\rho_{22} U_{x}\right) \\
\frac{\partial S}{\partial y}=\frac{\partial^{2}}{\partial t^{2}}\left(\rho_{11} V_{1}+\rho_{22} V_{y}\right) \\
\frac{\partial S}{\partial z}=\frac{\partial^{2}}{\partial t^{2}}\left(\rho_{11} W_{1}+\rho_{22} W_{z}\right)
\end{aligned}
$$

The displacement components of the solid porous materials are $\left(U_{1}, V_{1}, W_{1}\right)$, and $\left(U_{x}, V_{y}, W_{z}\right)$ are displacement components for the fluid saturated materials along $(x, y, z)$-directions. $S_{i j}^{M_{1}}, i, j=1,2,3$, and $S$ are stress components of solid and fluid part of the porous medium, respectively. The angular components $w_{x}, w_{y}$, and $w_{z}$ are given as 


$$
\begin{aligned}
& w_{x}=\frac{1}{2}\left(\frac{\partial w_{1}}{\partial y}-\frac{\partial v_{1}}{\partial z}\right) \\
& w_{y}=\frac{1}{2}\left(\frac{\partial u_{1}}{\partial z}-\frac{\partial w_{1}}{\partial x}\right) \\
& w_{z}=\frac{1}{2}\left(\frac{\partial v_{1}}{\partial x}-\frac{\partial u_{1}}{\partial y}\right)
\end{aligned}
$$

3.2. Density and Mass Equations. The stress vector of fluid part is $S=-f p$, where $f$ is the porosity of the medium and $p$ is the fluid pressure. We considered that there is no relative motion between liquid and solid part of the porous materials. The mass coefficients are considered as the inertial effects of the moving fluids and are directly related to the shear moduli of solid and fluid part of the porous medium. The mass coefficients $\rho_{11}, \rho_{12}$, and $\rho_{22}$ and densities of solid part $\rho_{s}$ and fluid part $\rho_{f}$ are related as $\rho_{11}+\rho_{12}=(1-f) \rho_{s}, \rho_{12}+\rho_{22}=f \rho_{f}$, and hence, the overall density of the porous medium is $\rho_{1}=\rho_{s}+f\left(\rho_{f}-\rho_{s}\right)$. Moreover, the mass coefficients are also restricted with the inequalities $\rho_{11}>0, \rho_{12} \leq 0, \rho_{22}>0$, and $\rho_{11} \rho_{22}-\rho_{12}^{2}>0$.

3.3. Displacement Equation of the Porous Medium. The direction of Love wave propagation is taken along $x$-axis. The nonvanishing displacement components of saturated porous medium are as follows.

Solid porous: $U_{1}=U_{1}(x, z, t)=0, V_{1}=V_{1}(x, z, t)$, and $W_{1}=W_{1}(x, z, t)=0$.

Fluid porous: $U_{1}^{*}=U_{1}^{*}(x, z, t)=0, V_{1}^{*}=V_{1}^{*}(x, z, t)$, and $W_{1}^{*}=W_{1}^{*}(x, z, t)=0$, and the nonvanishing equations of motion for solid and fluid saturated porous medium are

$$
\begin{aligned}
\frac{\partial S_{21}^{M_{1}}}{\partial x}+\frac{\partial S_{22}^{M_{1}}}{\partial y}+\frac{\partial S_{23}^{M_{1}}}{\partial z}-P\left(\frac{\partial w_{z}}{\partial x}\right) & =\frac{\partial^{2}}{\partial t^{2}}\left(\rho_{11} V_{1}+\rho_{12} V_{1}^{*}\right) \\
\frac{\partial S}{\partial y} & =\frac{\partial^{2}}{\partial t^{2}}\left(\rho_{11} V_{1}+\rho_{22} V_{1}^{*}\right)
\end{aligned}
$$

respectively. Using constitutive relationship of stress-strain in (6) and (7), we obtain

$$
\begin{aligned}
\left(N-\frac{P}{2}\right) \frac{\partial^{2} V_{1}}{\partial x^{2}}+L \frac{\partial^{2} V_{1}}{\partial z^{2}} & =\rho \frac{\partial^{2} V_{1}}{\partial t^{2}}, \\
\frac{\partial^{2}\left(\rho_{12} V_{1}+\rho_{22} V_{1}^{*}\right)}{\partial t^{2}} & =0,
\end{aligned}
$$

and $\rho_{12} V_{1}+\rho_{22} V_{1}^{*}=D^{\prime \prime}$; hence, $V_{1}^{*}=\left(D^{\prime \prime}-\rho_{12} V_{1}\right) / \rho_{22}, \rho$ $=\rho_{11}-\left(\rho_{12}^{2} / \rho_{22}\right)$, and $\left(\partial^{2} / \partial t^{2}\right)\left(\rho_{11} V_{1}+\rho_{12} V_{1}^{*}\right)=\rho\left(\partial^{2} V_{1}\right.$ $\left./ \partial t^{2}\right)$. The harmonic solution of $(8)$ is considered as

$$
V_{1}(x, z, t)=\phi(z) e^{i k(x-c t)}
$$

where $\phi(z)$ is stable function of $z, e^{i k(x-c t)}$ is temporary function of $x$ and time domain $t, k$ is the wavenumber, $c$ is the phase velocity, and $i=\sqrt{-1}$. From (8) and (10), we obtain

$$
\frac{\mathrm{d}^{2} \phi(z)}{\mathrm{d} z^{2}}+S_{1}^{2} \phi(z)=0,
$$

where $\quad S_{1}=k \sqrt{\left(\left[c^{2} d^{\prime}-(N-(P / 2))\right] / L\right)}=$ $k \sqrt{\beta_{1} d_{1}\left[\left(c^{2} / c_{1}^{2}\right)-\left(1-\beta_{2} / d_{1}\right)\right]}, \quad \beta_{1}=(N / L), \quad \beta_{2}=(P / 2 L)$, $c_{1}^{2}=\left(N / \rho^{M_{1}}\right), k$ is the wavenumber, and $d_{1}$ represents the porosity of incompressible porous medium. The porosity of the medium may be restricted as follows:

(i) $d_{1} \longrightarrow 1$, when the layer is a nonporous solid.

(ii) $d_{1} \longrightarrow 0$, when the layer is fluid.

(iii) $0<d_{1}<1$, when the layer is poro-elastic. The fundamental solution of (11) is obtained as

$$
\phi(z)=A_{1} \cos S_{1} z+A_{2} \sin S_{1} z .
$$

The displacement $V_{1}(x, z, t)$ in saturated solid porous medium is obtained as

$$
V_{1}(x, z, t)=\left(A_{1} \cos \left(S_{1} z\right)+A_{2} \sin \left(S_{1} z\right)\right) e^{i k(x-c t)},
$$

where $A_{1}$ and $A_{2}$ are arbitrary constants.

\section{Displacement Equation of the Exponentially Varying Orthotropic Medium}

The piezoelectric materials and fiber-reinforced composites are orthotropic in nature. Orthotropic materials have three mutually orthogonal planes of symmetry. Properties of material are independent of direction; such materials require 9 elastic constants in their constitutive matrices. The stress-strain relation for heterogeneous orthotropic material is given as

$$
\left[\begin{array}{c}
S_{11}^{M_{2}} \\
S_{22}^{M_{2}} \\
S_{33}^{M_{2}} \\
T_{23} \\
T_{13} \\
T_{12}
\end{array}\right]=\left[\begin{array}{cccccc}
\bar{M}_{11} & \bar{M}_{12} & \bar{M}_{13} & 0 & 0 & 0 \\
\bar{M}_{12} & \bar{M}_{22} & \bar{M}_{23} & 0 & 0 & 0 \\
\bar{M}_{13} & \bar{M}_{23} & \bar{M}_{33} & 0 & 0 & 0 \\
0 & 0 & 0 & \bar{M}_{44} & 0 & 0 \\
0 & 0 & 0 & 0 & \bar{M}_{55} & 0 \\
0 & 0 & 0 & 0 & 0 & \bar{M}_{66}
\end{array}\right]\left[\begin{array}{c}
\varepsilon_{11} \\
\varepsilon_{22} \\
\varepsilon_{33} \\
\gamma_{23} \\
\gamma_{13} \\
\gamma_{12}
\end{array}\right] e^{\bar{\alpha} z} .
$$

where $S_{11}^{M_{2}}, S_{22}^{M_{2}}$, and $S_{33}^{M_{2}}$ are normal stresses, $M_{2}$ stands for orthotropic medium, $T_{23}, T_{13}$, and $T_{12}$ are shear stresses, $\varepsilon_{11}$, $\varepsilon_{22}$, and $\varepsilon_{33}$ are normal strains, $\gamma_{23}, \gamma_{13}$, and $\gamma_{12}$ are shear strain, and $\bar{M}_{i j}$ are elastic constants of orthotropic medium. The relation between Young's moduli $\left(E_{j}, j=1,2,3\right)$ and Poisson's ratios $\left(v_{i j}, i, j=1,2,3\right)$ is $v_{i j} / E_{i}=v_{j i} / E_{j}, i, j=$ $1,2,3$. Hence, the elastic constants $\bar{M}_{i j}$ can be presented in terms of independent elastic constants as 


$$
\begin{aligned}
& \bar{M}_{11}=\frac{E_{1}\left(\nu_{23} \nu_{31}-1\right)}{v_{12}\left(\nu_{21}+v_{23} \nu_{31}\right)+v_{23} \nu_{31}+v_{13}\left(\nu_{31}+v_{21} \nu_{32}\right)-1}, \\
& \bar{M}_{12}=-\frac{E_{1}\left(\nu_{21}+v_{23} v_{31}\right)}{v_{12}\left(v_{21}+v_{23} \nu_{31}\right)+v_{23} \nu_{31}+v_{13}\left(v_{31}+v_{21} v_{32}\right)-1}, \\
& \bar{M}_{13}=-\frac{E_{1}\left(\nu_{31}+\nu_{21} \nu_{32}\right)}{v_{12}\left(\nu_{21}+\nu_{23} \nu_{31}\right)+\nu_{23} \nu_{31}+\nu_{13}\left(\nu_{31}+v_{21} \nu_{32}\right)-1}, \\
& \bar{M}_{22}=\frac{E_{2}\left(\nu_{13} \nu_{31}-1\right)}{v_{12}\left(\nu_{21}+v_{23} \nu_{31}\right)+v_{23} \nu_{31}+v_{13}\left(\nu_{31}+v_{21} v_{32}\right)-1}, \\
& \bar{M}_{23}=-\frac{E_{2}\left(\nu_{32}+v_{12} \nu_{31}\right)}{v_{12}\left(\nu_{21}+v_{23} \nu_{31}\right)+v_{23} \nu_{31}+v_{13}\left(\nu_{31}+v_{21} v_{32}\right)-1} \text {, } \\
& \bar{M}_{33}=\frac{E_{3}\left(\nu_{12} \nu_{21}-1\right)}{v_{12}\left(\nu_{21}+\nu_{23} \nu_{31}\right)+\nu_{23} \nu_{31}+\nu_{13}\left(\nu_{31}+\nu_{21} \nu_{32}\right)-1}, \\
& \bar{M}_{44}=G_{23}, \bar{M}_{55}=G_{13}, \bar{M}_{66}=G_{12} \text {, }
\end{aligned}
$$

$$
V_{2}(x, z, t)=\bar{\phi}(z) e^{i k(x-c t)},
$$

where $G_{i j}, i, j=1,2,3$, are shear moduli of orthotropic medium.

The differential forms of the governing equations of motion for orthotropic materials are

$$
\begin{aligned}
& \frac{\partial S_{11}^{M_{2}}}{\partial x}+\frac{\partial T_{12}}{\partial y}+\frac{\partial T_{13}}{\partial z}=\rho^{M_{2}} \frac{\partial^{2} U_{2}}{\partial t^{2}} \\
& \frac{\partial T_{12}}{\partial x}+\frac{\partial S_{22}^{M_{2}}}{\partial y}+\frac{\partial T_{23}}{\partial z}=\rho^{M_{2}} \frac{\partial^{2} V_{2}}{\partial t^{2}} \\
& \frac{\partial T_{13}}{\partial x}+\frac{\partial T_{23}}{\partial y}+\frac{\partial S_{33}^{M_{2}}}{\partial z}=\rho^{M_{2}} \frac{\partial^{2} W_{2}}{\partial t^{2}}
\end{aligned}
$$

Using constitutive relations (14) and standard Love wave propagation conditions in the governing equations of motion $(22 \mathrm{a})-(22 \mathrm{c})$, we obtain

$$
\begin{gathered}
\bar{M}_{66} \frac{\partial^{2} U_{2}}{\partial x^{2}}+\bar{M}_{44} \frac{\partial^{2} U_{2}}{\partial z^{2}}+\alpha \bar{M}_{44} \frac{\partial U_{2}}{\partial z}=\rho^{M_{2}} \frac{\partial^{2} U_{2}}{\partial t^{2}}, \\
\bar{M}_{11} \frac{\partial^{2} V_{2}}{\partial x^{2}}+\bar{M}_{55} \frac{\partial^{2} V_{2}}{\partial z^{2}}+\alpha \bar{M}_{55} \frac{\partial V_{2}}{\partial z}=\rho^{M_{2}} \frac{\partial^{2} V_{2}}{\partial t^{2}}, \\
\bar{M}_{55} \frac{\partial^{2} W_{2}}{\partial x^{2}}+\bar{M}_{33} \frac{\partial^{2} W_{2}}{\partial z^{2}}+\alpha \bar{M}_{33} \frac{\partial W_{2}}{\partial z}=\rho^{M_{2}} \frac{\partial^{2} W_{2}}{\partial t^{2}} .
\end{gathered}
$$

The nonvanishing displacement of orthotropic medium will occur along $y$-direction only; i.e., $U_{2}=U_{2}(x, z, t)=0$, $V_{2}=V_{2}(x, z, t)$, and $W_{2}=W_{2}(x, z, t)=0$. Hence, the displacement along $y$-direction is the general solution of (23b).

The standard form of the solution of the harmonic wave is assumed as and using the above relation in (23b), we have

$$
\bar{M}_{44} \frac{\mathrm{d}^{2} \bar{\phi}(z)}{\mathrm{d} z^{2}}+\alpha \frac{\mathrm{d} \bar{\phi}(z)}{\mathrm{d} z}+\frac{k^{2}\left(\rho^{M_{2}} c^{2}-\bar{M}_{66}\right)}{\bar{M}_{44}} \bar{\phi}(z)=0 .
$$
as

The general solution of (25) can be obtained analytically

$$
\bar{\phi}(z)=A_{3} e^{S_{2}}+A_{4} e^{S_{3}}
$$

where $A_{3}$ and $A_{4}$ are arbitrary constants and

$$
\begin{gathered}
S_{2}=\frac{-\bar{\alpha}}{2}-\frac{\sqrt{\bar{\alpha}^{2}-4 k^{2}\left(\rho^{M_{2}} c^{2}-\bar{M}_{66} / \bar{M}_{44}\right)}}{2}, \\
S_{3}=\frac{-\bar{\alpha}}{2}+\frac{\sqrt{\bar{\alpha}^{2}-4 k^{2}\left(\rho^{M_{2}} c^{2}-\bar{M}_{66} / \bar{M}_{44}\right)}}{2} .
\end{gathered}
$$

The bounded displacement $\left(V_{2}(x, z, t)\right)$ in the semiinfinite medium may be obtained by assuming $A_{4}=0$ when $z \longrightarrow 0$ so that

$$
V_{2}(x, z, t)=A_{3} \exp \left(\frac{-\bar{\alpha}}{2}-\frac{\sqrt{\bar{\alpha}^{2}-4 k^{2}\left(\rho^{M_{2}} c^{2}-\bar{M}_{66} / \bar{M}_{44}\right)}}{2}\right) z .
$$

The complete displacement in exponentially varying orthotropic semi-infinite medium is obtained in (20).

\section{Continuity Conditions for Model-I and Model-II}

The surface of saturated porous medium (M-I) is assumed as irregular rigid mountain surface in Model-I and soft irregular mountain surface in Model-II, and the continuity equations are as follows. 
5.1. Continuity at the Surface $z=\lambda_{1}(x)-H$.

(1) Model-I: due to high rigidity of the surface, the displacement of solid porous medium is vanishing at $z=\lambda_{1}(x)-H$; i.e., $V_{1}(x, z, t)=0$ at $z=\lambda_{1}(x)-H$

(2) Model-II: in this case, the surface of saturated porous medium is assumed as soft irregular mountain surface; i.e., $S_{23}^{M_{1}}-\lambda_{1}^{\prime} S_{12}^{M_{1}}=0$ at $z=\lambda_{1}(x)-H$

5.2. Continuity at the Interface $z=0$. The continuity equations at the contact interface of M-I and M-II for both models are

(1) The continuity of the stress components at the contact interface of M-I and M-II requires $S_{23}^{M_{1}}=S_{23}^{M_{2}}$ at $z=0$

(2) The continuity of the displacement of M-I and M-II requires $V_{1}(x, z, t)=V_{2}(x, z, t)$ at $z=0$

\section{Dispersion Relation}

6.1. Phase Velocity and Displacement Relation for Model-I. Using continuity conditions of Model-I in displacement equations (13) and (29), we obtain the following phase velocity equations for Model-I:

$$
\begin{aligned}
\cos S_{1}\left(\lambda_{1}-H\right) A_{1}+\sin S_{1}\left(\lambda_{1}-H\right) A_{2} & =0, \\
L S_{1} A_{2}+\bar{M}_{44} S_{2} A_{3} & =0, \\
A_{1}-A_{3} & =0 .
\end{aligned}
$$

The generalized dispersion relation of Love wave under rigid mountain surfaces may be obtained by eliminating arbitrary constants $A_{1}, A_{2}$, and $A_{3}$ from (30a)-(30c) as

$$
\left|\begin{array}{ccc}
\cos S_{1}\left(\lambda_{1}-H\right) & \sin S_{1}\left(\lambda_{1}-H\right) & 0 \\
0 & L S_{1} & \bar{M}_{44} S_{2} \\
1 & 0 & -1
\end{array}\right|=0 .
$$

The generalized nondimensional form of the dispersion relation in rigid mountain surface may be obtained as

$$
\begin{aligned}
& \cot \left(k H\left(\frac{a}{H}\right) \cos \left(b H\left(\frac{x}{H}\right)\right)-k H\right) \sqrt{\beta_{1} d_{1}\left(\frac{c^{2}}{c_{1}^{2}}-\frac{1-\beta_{2}}{d_{1}}\right)} \\
& =\frac{\bar{M}_{44}}{L} \frac{-(\bar{\alpha} / 2 k)-\sqrt{(\bar{\alpha} / 2 k)^{2}-\left(c^{2} / c_{2}^{2}\right)+\left(\bar{M}_{66} / \bar{M}_{44}\right)}}{\sqrt{\beta_{1} d_{1}\left(\left(c^{2} / c_{1}^{2}\right)-\left(\left(1-\beta_{2}\right) / d_{1}\right)\right)}} .
\end{aligned}
$$

\subsection{Classical Dispersion Relation for Model-I}

6.2.1. Case-I. Suppose the surface of the saturated porous medium is plain, i.e., $\lambda_{1}(x)=0$ and $z=-H$. In this case, the generalized form of the dispersion relation may be reduced to

$$
\cot \left(-k H \sqrt{\beta_{1} d_{1}\left(\frac{c^{2}}{c_{1}^{2}}-\frac{1-\beta_{2}}{d_{1}}\right)}\right)=\frac{\bar{M}_{44}}{L} \frac{-(\bar{\alpha} / 2 k)-\sqrt{(\bar{\alpha} / 2 k)^{2}-\left(c^{2} / c_{2}^{2}\right)+\left(\bar{M}_{66} / \bar{M}_{44}\right)}}{\sqrt{\beta_{1} d_{1}\left(\left(c^{2} / c_{1}^{2}\right)-\left(1-\beta_{2} / d_{1}\right)\right)}} .
$$

6.2.2. Case-II. If the superficial layer is initially a stress-free, homogeneous, isotropic, and nonporous type material, i.e., $d_{1}=1, N=L=\mu_{1}$, and $(P / 2 L)=0$. The generalized dispersion may be reduced to

$$
\cot \left(k H \sqrt{\left(\frac{c^{2}}{c_{1}^{2}}-1\right)}\right)=\frac{\bar{M}_{44}}{\mu_{1}} \frac{(\bar{\alpha} / 2 k)+\sqrt{(\bar{\alpha} / 2 k)^{2}-\left(c^{2} / c_{2}^{2}\right)+\left(\bar{M}_{66} / \bar{M}_{44}\right)}}{\sqrt{\left(\left(c^{2} / c_{1}^{2}\right)-1\right)}} .
$$


6.2.3. Case-III. If the semi-infinite medium is a homogeneous, isotropic-type material, i.e., $\bar{M}_{44}=\bar{M}_{66}=\mu_{2}$ and $\bar{\alpha}=0$, the generalized dispersion may reduce to classical dispersion relation of Love wave [1]:

$$
\cot \left(k H \sqrt{\left(\frac{c^{2}}{c_{1}^{2}}-1\right)}\right)=\frac{\mu_{2}}{\mu_{1}} \frac{\sqrt{1-\left(c^{2} / c_{2}^{2}\right)}}{\sqrt{\left(c^{2} / c_{1}^{2}\right)-1}} .
$$

6.3. Phase Velocity and Displacement Relation for Model-II. The phase velocity equations of Model-II are obtained as

$$
\begin{gathered}
-\left(L S_{1} \sin \left\{S_{1}\left(\lambda_{1}-H\right)\right\}+i k N \frac{d \lambda_{1}}{d z} \cos \left\{S_{1}\left(\lambda_{1}-H\right)\right\}\right) A_{1}, \\
+\left(L S_{1} \cos \left\{S_{1}\left(\lambda_{1}-H\right)\right\}-i k N \frac{d \lambda_{1}}{d z} \sin \left\{S_{1}\left(\lambda_{1}-H\right)\right\}\right) A_{2}=0 \\
L S_{1} A_{2}+\bar{M}_{44} S_{2} A_{3}=0
\end{gathered}
$$

$$
A_{1}-A_{3}=0 .
$$

The generalized dispersion relation of Love wave under soft mountain surfaces may be obtained by eliminating arbitrary constants $A_{1}, A_{2}$, and $A_{3}$ from (36a)-(36c) as

$$
\left|\begin{array}{ccc}
a_{11} & a_{12} & 0 \\
0 & L S_{1} & \bar{M}_{44} S_{2} \\
1 & 0 & -1
\end{array}\right|=0
$$

where $a_{11}=-\left(L S_{1} \sin \left\{S_{1}\left(\lambda_{1}-H\right)\right\}+i k N\left(d \lambda_{1} / d z\right) \cos \left\{S_{1}\right.\right.$ $\left.\left.\left(\lambda_{1}-H\right)\right\}\right)$ and $a_{12}=\left(L S_{1} \cos \left\{S_{1}\left(\lambda_{1}-H\right)\right\}-i k N\left(\mathrm{~d} \lambda_{1} / \mathrm{d} z\right)\right.$ $\left.\sin \left\{S_{1}\left(\lambda_{1}-H\right)\right\}\right)$.

The complex form of the generalized dispersion relation of Love wave under soft mountain surfaces is obtained as

$$
\begin{aligned}
& {\left[L^{2} S_{1}^{2} \sin \left\{S_{1}\left(\lambda_{1}-H\right)\right\}+L S_{1} \bar{M}_{44} S_{2} \cos \left\{S_{1}\left(\lambda_{1}-H\right)\right\}\right]} \\
& \quad+i\left[\operatorname{LNS}_{1} \frac{\mathrm{d} \lambda_{1}}{\mathrm{~d} z} \cos \left\{S_{1}\left(\lambda_{1}-H\right)\right\}-\bar{M}_{44} N S_{2} \frac{\mathrm{d} \lambda_{1}}{\mathrm{~d} z} \sin \left\{S_{1}\left(\lambda_{1}-H\right)\right\}\right]=0 .
\end{aligned}
$$

The dispersion relation of Love wave propagation under soft mountain surface may be obtained by equating real part of (28) as

$$
\left[-L^{2} S_{1}^{2} \sin \left\{S_{1}\left(H-\lambda_{1}\right)\right\}+L S_{1} \bar{M}_{44} S_{2} \cos \left\{S_{1}\left(H-\lambda_{1}\right)\right\}\right]=0,
$$

and the generalized form is

$$
\begin{aligned}
& \left.\tan \{k H(1-(a / H)) \cos (b H(x / H))) \sqrt{\beta_{1} d_{1}\left(\frac{c^{2}}{c_{1}^{2}}-\frac{1-\beta_{2}}{d_{1}}\right)}\right\} \\
& =\frac{\bar{M}_{44}}{L} \frac{(\bar{\alpha} / 2 k)+\sqrt{((\bar{\alpha} / 2 k))^{2}-\left(c^{2} / c_{2}^{2}\right)+\left(\bar{M}_{66} / \bar{M}_{44}\right)}}{\sqrt{\beta_{1} d_{1}\left(\left(c^{2} / c_{1}^{2}\right)-\left(\left(1-\beta_{2}\right) / d_{1}\right)\right)}} .
\end{aligned}
$$

\subsection{Classical Dispersion Relation for Model-II}

6.4.1. Case-I. Suppose the surface of the saturated porous medium is a plain surface (regular surface), i.e., $\lambda_{1}(x)=0$ and $z=-H$. In this case, the generalized form of the dispersion may be obtained as

$$
\tan \left\{k H\left(\sqrt{\beta_{1} d_{1}\left(\frac{c^{2}}{c_{1}^{2}}-\frac{1-\beta_{2}}{d_{1}}\right)}\right)\right\}=\frac{\bar{M}_{44}}{L} \frac{(\bar{\alpha} / 2 k)+\sqrt{(\bar{\alpha} / 2 k)^{2}-\left(c^{2} / c_{2}^{2}\right)+\left(\bar{M}_{66} / \bar{M}_{44}\right)}}{\sqrt{\beta_{1} d_{1}\left(\left(c^{2} / c_{1}^{2}\right)-\left(\left(1-\beta_{2}\right) / d_{1}\right)\right)}}
$$

6.4.2. Case-II. If the superficial layer is initially a stress-free, homogeneous, isotropic, and nonporous-type material, i.e., $d_{1}=1, N=L=\mu_{1}$, and $P / 2 L=0$, the generalized dispersion may be reduced to the following form: 
TABLE 1: General data of parameters for graphical results in mountain surface.

\begin{tabular}{lcccccccc}
\hline Parameter & Figure 2 & Figure 3 & Figure 4 & Figure 5 & Figure 6 & Figure 7 & Figure 8 & Figure 9 \\
\hline Initial stress $P / 2 L$ & - & 0.2 & 0.2 & 0.2 & - & 0.2 & 0.2 & 0.2 \\
Porosity $\left(d_{1}\right)$ & 0.7 & 0.7 & 0.7 & - & 0.7 & 0.7 & 0.7 & - \\
Heterogeneity $(\alpha / k)$ & 0.2 & 0.2 & - & 0.2 & 0.2 & 0.2 & 0.2 \\
Velocity $\left(c_{2} / c_{2}^{2}\right)$ & 0.1 & 0.1 & 0.1 & 0.1 & 0.1 & 0.1 & 0.1 \\
$x / H$ & 0.10 & - & 0.10 & 0.10 & 0.10 & - & 0.10 \\
$b H$ & 1.20 & - & 1.20 & 1.20 & 0.0 & - & 0.1 \\
$a / H$ & 0.02 & 0.02 & 0.02 & 0.02 & 0.02 & 0.02 & 0.02 \\
\hline
\end{tabular}

TABLe 2: General data for the proposed Earth model.

\begin{tabular}{|c|c|c|}
\hline Materials & Elastic constant & Value \\
\hline Porous & $\begin{array}{l}\text { Rigidities } \\
\text { Density } \\
\text { Density } \\
\text { Porosity }\end{array}$ & $\begin{aligned} N & =0.2774 \times 10^{10} \mathrm{~N} / \mathrm{m}^{2} \\
L & =0.1387 \times 10^{10} \mathrm{~N} / \mathrm{m}^{2} \\
\rho_{11} & =1.926137 \times 10^{3} \mathrm{~kg} / \mathrm{m}^{3} \\
\rho_{22} & =0.215337 \times 10^{3} \mathrm{~kg} / \mathrm{m}^{3}\end{aligned}$ \\
\hline \multirow{3}{*}{ Orthotropic } & $\begin{array}{l}\text { Rigidities } \\
\text { Density }\end{array}$ & $\begin{aligned} \bar{M}_{66} & =3.99 \times 10^{10} \mathrm{~N} / \mathrm{m}^{2} \\
\bar{M}_{44} & =5.82 \times 10^{10} \mathrm{~N} / \mathrm{m}^{2} \\
\rho^{M_{2}} & =1.6 \times 10^{3} \mathrm{~kg} / \mathrm{m}^{2}\end{aligned}$ \\
\hline & Young's moduli & $\begin{array}{l}E_{1}=33.83 \mathrm{GPa} \\
E_{2}=47.64 \mathrm{GPa} \\
E_{3}=49.09 \mathrm{GPa} \\
G_{12}=15.8 \mathrm{GPa}\end{array}$ \\
\hline & Shear moduli & $\begin{aligned} G_{13} & =16.2 \mathrm{GPa} \\
G_{23} & =19.4 \mathrm{GPa} \\
v_{12} & =0.11 \mathrm{GPa} \\
v_{13} & =0.05 \mathrm{GPa} \\
v_{23} & =0.21 \mathrm{GPa}\end{aligned}$ \\
\hline
\end{tabular}

$$
\cot \left(k H \sqrt{\left(\frac{c^{2}}{c_{1}^{2}}-1\right)}\right)=\frac{\bar{M}_{44}}{\mu_{1}} \frac{(\bar{\alpha} / 2 k)+\sqrt{(\bar{\alpha} / 2 k)^{2}-\left(c^{2} / c_{2}^{2}\right)+\left(\bar{M}_{66} / \bar{M}_{44}\right)}}{\sqrt{\left(\left(c^{2} / c_{1}^{2}\right)-1\right)}} .
$$

6.4.3. Case-III. If the semi-infinite medium is a homogeneous, isotropic-type material, i.e., $\bar{M}_{44}=\bar{M}_{66}=\mu_{2}$ and $\bar{\alpha}=0$, the generalized dispersion relation is reduced to classical dispersion relation of Love wave [1]:

$$
\tan \left(k H \sqrt{\left(\frac{c^{2}}{c_{1}^{2}}-1\right)}\right)=\frac{\mu_{2}}{\mu_{1}} \frac{\sqrt{1-\left(c^{2} / c_{2}^{2}\right)}}{\sqrt{\left(c^{2} / c_{1}^{2}\right)-1}} .
$$

\section{Numerical Computations and Discussion}

The numerical values [4] of the elastic constants are demonstrated in Tables 1 and 2 .

In this paper, the dispersion relation of the Love wave has been derived analytically for both models. The obtained dispersion relation is well-matched with the standard dispersion relation of the Love wave.

The following conclusions can be drawn from the presented parametric study.

Figures 2 and 3 reflect the effect of initial stress $(P / 2 L)$ on the phase velocity of the Love wave under the rigid mountain surface and soft mountain surface, respectively. It has been noticed that the phase velocity decreases in both cases as
$(P / 2 L)$ increases. The impact of $(P / 2 L)$ on phase velocity $\left(c^{2} / c_{1}^{2}\right)$ is visible for the wavenumbers $k H=1.2$ to 1.9 in case of rigid mountain surface, as shown in Figure 2, but the impact of the initial stress is visible for the large range of the wavenumber $(k H=1.8$ to 2.8$)$ under the soft mountain surface, as mentioned in Figure 3. Hence, the effect of rigid and soft mountain surface is considerable during Love wave propagation in Earth medium.

Figures 4 and 5 demonstrate the effect of rigid and soft irregular mountain surfaces on the phase velocity of Love wave. The phase velocity of the Love wave decreases as the amplitude of the irregularity increases. It can be seen that the effect of the irregular surfaces on the phase velocity remains the same for both models, but the rigidity of the mountain surface compressed the effect for small range of the wavenumber $(k H)$.

Figures 6 and 7 depict the influence of heterogeneity of the orthotropic medium on the phase velocity of Love wave propagation under rigid and soft mountain surfaces, respectively. It is noticed that the phase velocity of the wave decreases as the heterogeneity $(\alpha / k=.1, .5, .9)$ of the materials increases for Model-I. However, the phase velocity of the wave increases as the heterogeneity $(\alpha / k=$ $.1, .5, .9)$ of the medium increases for Model-II. It has been 


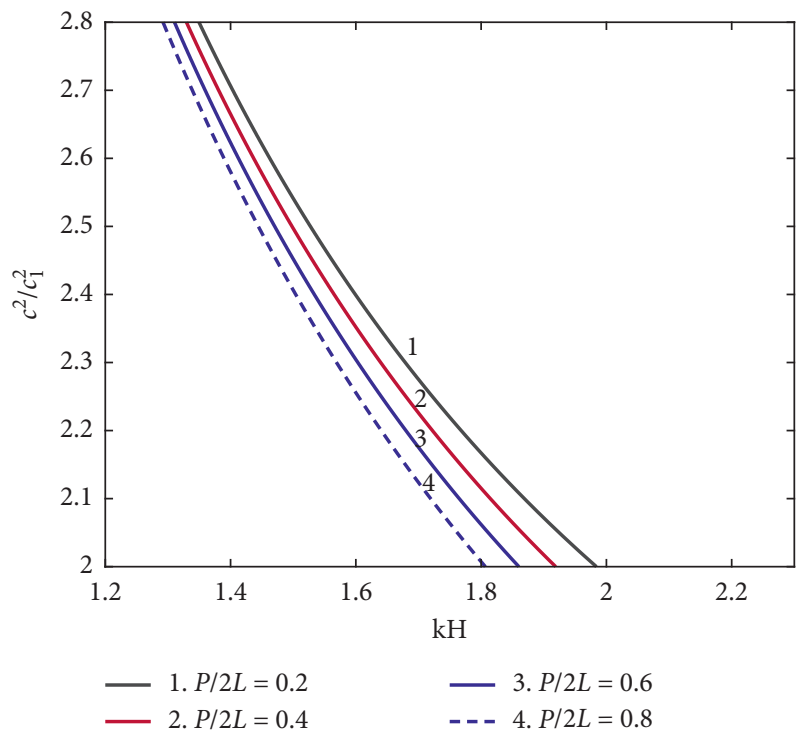

FIGURE 2: Model-II: medium of the propagation under soft mountain surface.

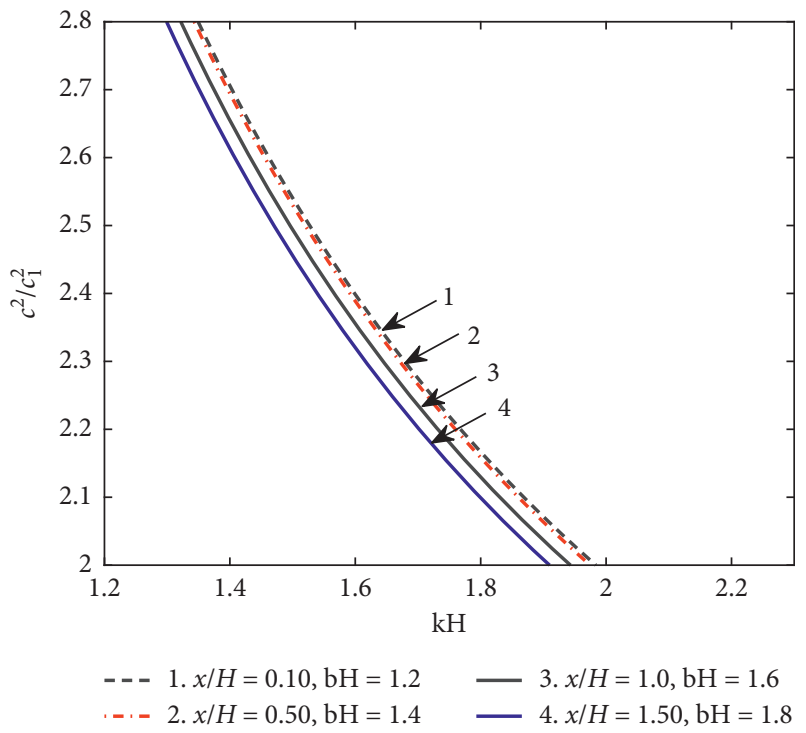

FIGURE 3: Medium of the Love wave propagation.

noticed that the rigidity of the mountains vomits the effect of heterogeneity on the phase velocity of Love wave. Influence of the irregularity is visible for the small range of the wavenumber under rigid mountain surface, as demonstrated in Figure 6. However, it is visible for large range of the wavenumber in the case of soft mountain surface, as shown in Figure 7.
Figures 8 and 9 reflect the effect of porosity of the saturated porous medium on the phase velocity of the Love wave for Model-I and Model-II, respectively. Generally, the porosity of the medium decreases the phase velocity of the Love wave in both models. The influence of the porosity is visible for small wavenumbers under Model-I, but it is visible for large range of wavenumber under Model-II. 


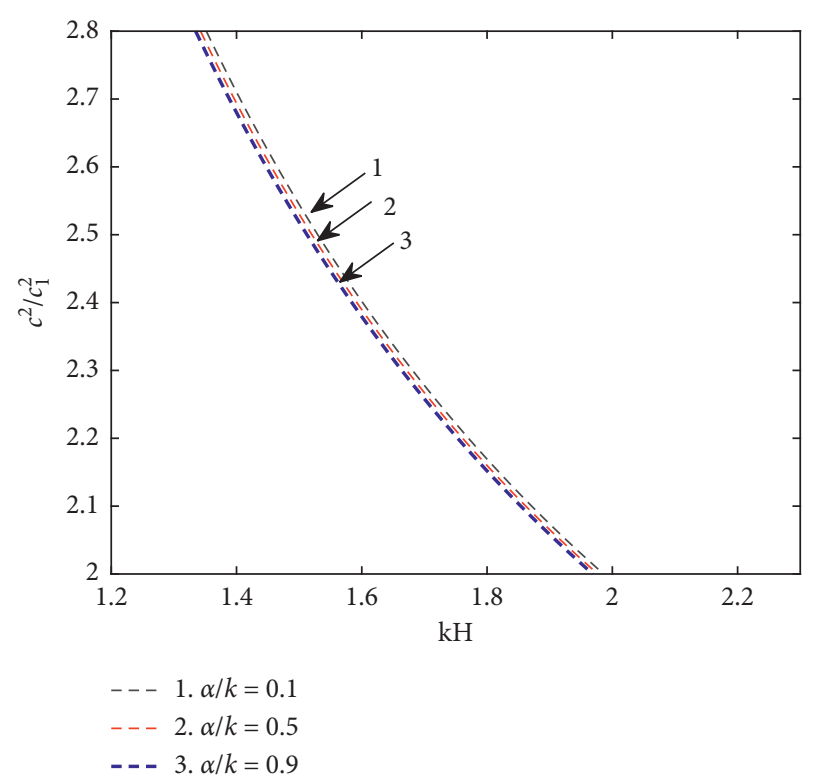

Figure 4: Model-I: effect of the initial stress $(P / 2 L)$ under rigid mountain surface.

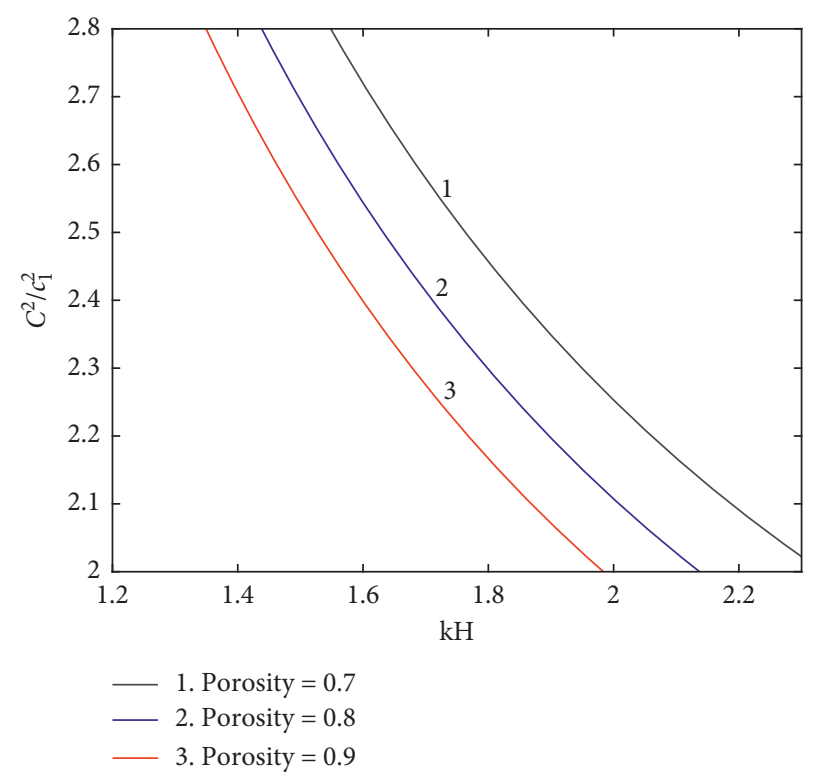

Figure 5: Model-II: effect of the initial stress $(P / 2 L)$ under soft mountain surface.
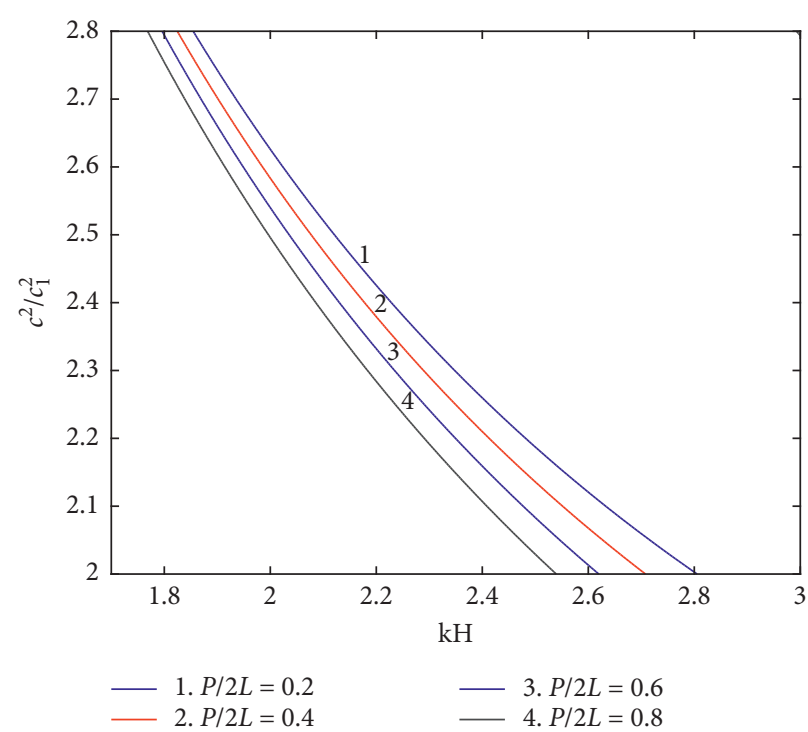

Figure 6: Model-I: effect of the irregularity parameters $(x / H)$ and $(b H)$ under rigid mountain surface.

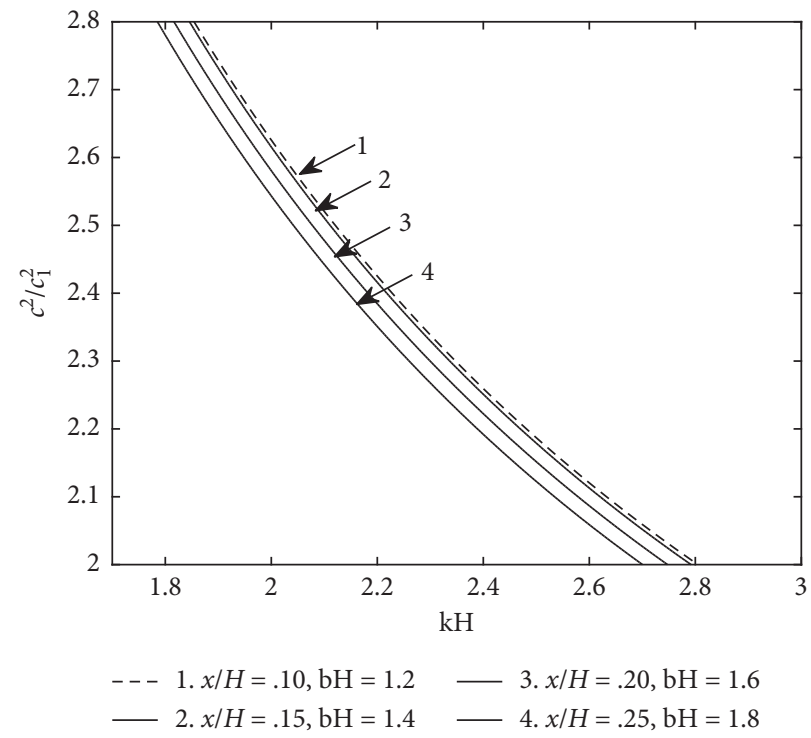

FIgURE 7: Model-II: effect of the irregularity parameters $(x / H)$ and $(b H)$ under soft mountain surface. 


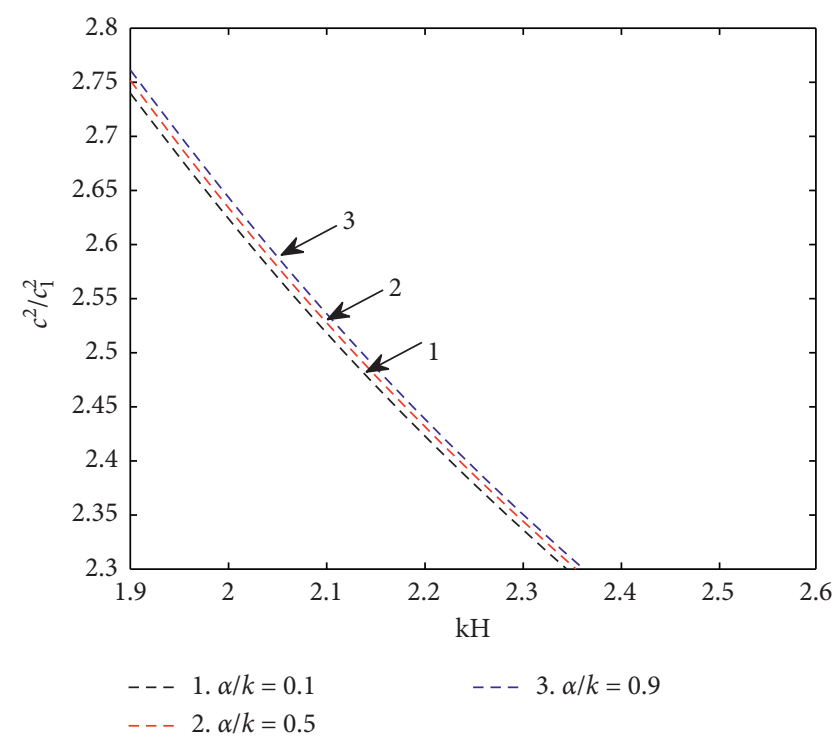

FIGURE 8: Model-I: effect of the heterogeneity parameters $(\alpha / k)$ under rigid mountain surface.

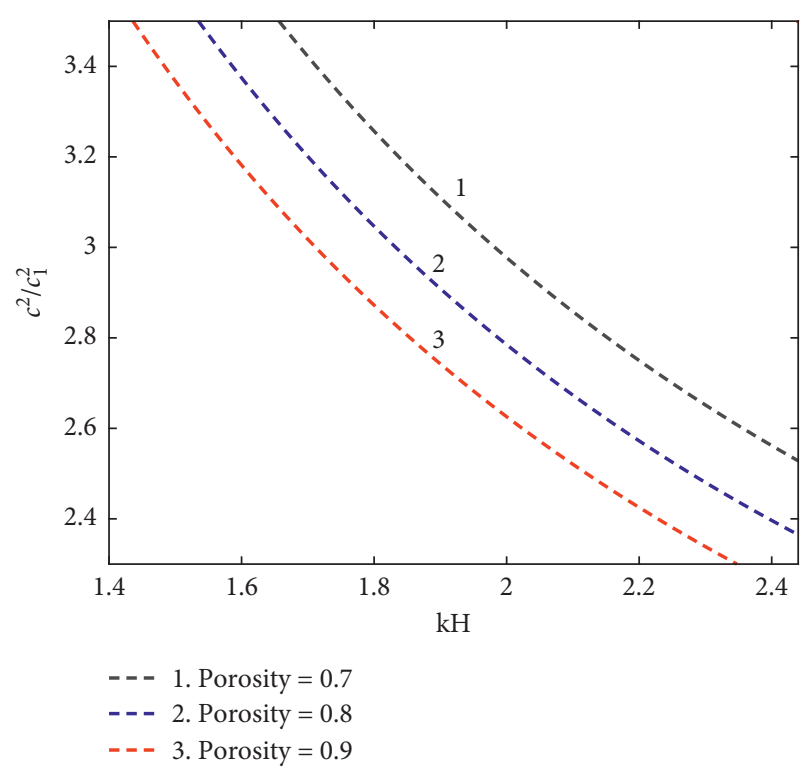

Figure 9: Model-II: effect of the heterogeneity parameters $(\alpha / k)$ under soft mountain surface.

\section{Conclusions}

The present study exposes the effect of rigid (Model-I) and soft (Model-II) mountain surfaces on the phase velocity of the Love wave propagation. The parametric effects on the phase velocity can be concluded as follows:

(1) The phase velocity of the Love wave decreases in the presence of initial stress $(P / 2 L)$. It has been noticed that the effect of initial stress is visible in two different ranges of the frequency in two different models, as shown in Figures 2 and 3.

(2) The irregular surface (mountain surface) of the superficial layer decreases the phase velocity in both rigid (Model-I) and soft (Model-II) mountain surfaces as demonstrated in Figures 4 and 5.

(3) It has been observed that the heterogeneity of the half-space affects the phase velocity in both models. The phase velocity decreases as the value of $\alpha / k$ increases. The moderate effect of the heterogeneity on the phase velocity has been recorded in both cases due to irregular surface, as depicted in Figures 6 and 7.

(4) The significant effect of the porosity of the fluid saturated porous medium on the phase velocity has been recorded in Figures 8 and 9. The phase velocity decreases as the porosity $\left(d_{1}\right)$ of the medium increases.

It has been observed that the parametric effects on the phase velocity of Love wave remains constant in both cases. However, the different ranges of the frequency are recorded for two different models.

\section{Data Availability}

All data, models, and code generated or used during the study are included within the article.

\section{Conflicts of Interest}

The authors declare that there are no conflicts of interest regarding the publication of this paper.

\section{Acknowledgments}

The authors convey their sincere thanks to the TIET, Patiala, for providing the best facilities, and the authors would like to acknowledge the financial support of Taif University researchers, supporting project no. TURSP-2020/162, Taif University, Saudi Arabia.

\section{References}

[1] A. E. H. Love, Some Problems of Geodynamics, Cambridge University Press, Cambridge, UK, 1911.

[2] M. A. Biot, Mechanics of Incremental Deformation, John Willey and Sons, New York, NY, USA, 1965.

[3] M. Ewing, W. Jardetzky, and F. Press, Elastic Waves in Layered Media, McGraw-Hill Book Company, New York, NY, USA, 1957.

[4] D. Gubbins, Seismology and Plate Tectonics, Cambridge University Press, Cambridge, UK, 1990.

[5] A. M. Abd-Alla, S. R. Mahmoud, S. M. Abo-Dahab, and M. I. Helmy, "Influences of rotation, magnetic field, initial stress, and gravity on Rayleigh waves in a homogeneous orthotropic elastic half-space," Applied Mathematical Sciences, vol. 4, no. 2, pp. 91-108, 2010.

[6] S. Gupta, A. Chattopadhyay, and D. K. Majhi, "Effect of initial stress on propagation of Love waves in an anisotropic porous layer," Solid Mechanics, vol. 2, pp. 50-62, 2010.

[7] S. M. Ahmed and S. M. Abo-Dahab, "Propagation of Love waves in an orthotropic granular layer under initial stress overlying a semi-infinite granular medium," Journal of $\mathrm{Vi}$ bration and Control, vol. 16, no. 12, pp. 1845-1858, 2010. 
[8] S. K. Vishwakarma, S. Gupta, and D. K. Majhi, "Influence of rigid boundary and initial stress on the propagation of Love wave," Applied Mathematics, vol. 02, no. 05, pp. 586-594, 2011.

[9] R. Ogden and B. Singh, "Propagation of waves in an incompressible transversely isotropic elastic solid with initial stress: Biot revisited," Journal of Mechanics of Materials and Structures, vol. 6, no. 1-4, pp. 453-477, 2011.

[10] A. M. Abd-Alla, S. M. Abo-Dahab, and T. A. Al-Thamali, "Love waves in a non-homogeneous orthotropic magnetoelastic layer under initial stress overlying a semi-infinite medium," Journal of Computational and Theoretical Nanoscience, vol. 10, no. 1, pp. 10-18, 2013.

[11] S. Gupta, D. K. Majhi, S. Kundu, and S. K. Vishwakarma, "Propagation of Love waves in non-homogeneous substratum over initially stressed heterogeneous half-space," Applied Mathematics and Mechanics, vol. 34, no. 2, pp. 249-258, 2013.

[12] R. W. Ogden and B. Singh, "The effect of rotation and initial stress on the propagation of waves in a transversely isotropic elastic solid," Wave Motion, vol. 51, no. 7, pp. 1108-1126, 2014.

[13] R. Kakar and S. Kakar, "S-wave propagating in an anisotropic inhomogeneous elastic medium under the influence of gravity, initial stress, electric and magnetic field," Theoretical and Applied Mechanics, vol. 41, no. 2, pp. 141-157, 2014.

[14] J. Pal and A. P. Ghorai, "Propagation of Love wave in sandy layer under initial stress above anisotropic porous half-space under gravity," Transport in Porous Media, vol. 109, no. 2, pp. 297-316, 2015.

[15] M. Shams, "Effect of initial stress on Love wave propagation at the boundary between a layer and a half-space," Wave Motion, vol. 65 , pp. 92-104, 2016.

[16] N. T. Nam, J. Merodio, R. W. Ogden, and P. C. Vinh, "The effect of initial stress on the propagation of surface waves in a layered half-space," International Journal of Solids and Structures, vol. 89, pp. 88-100, 2016.

[17] K. Ejaz and M. Shams, "Love waves in compressible elastic materials with a homogeneous initial stress," Mathematics and Mechanics of Solids, vol. 24, no. 8, pp. 2576-2590, 2019.

[18] S. Kundu, M. Maity, D. K. Pandit, and S. Gupta, "Effect of initial stress on the propagation and attenuation characteristics of Rayleigh waves," Acta Mechanica, vol. 230, no. 1, pp. $67-85,2019$.

[19] L. Tochhawng and S. Singh, "Effect of initial stresses on the elastic waves in transversely isotropic thermoelastic materials," Engineering Reports, vol. 2, pp. 1-14, 2020.

[20] L.-L. Ke, Y.-S. Wang, and Z.-M. Zhang, "Propagation of Love waves in an inhomogeneous fluid saturated porous layered half-space with properties varying exponentially," Journal of Engineering Mechanics, vol. 131, no. 12, pp. 1322-1328, 2005.

[21] A. P. Ghorai, S. K. Samal, and N. C. Mahanti, "Love waves in a fluid-saturated porous layer under a rigid boundary and lying over an elastic half-space under gravity," Applied Mathematical Modelling, vol. 34, no. 7, pp. 1873-1883, 2010.

[22] W. Chen, T. Xia, and W. Hu, "A mixture theory analysis for the surface-wave propagation in an unsaturated porous medium," International Journal of Solids and Structures, vol. 48, no. 16-17, pp. 2402-2412, 2011.

[23] R. Kumar and R. Kumar, "Analysis of wave motion at the boundary surface of orthotropic thermoelastic material with voids and isotropic elastic half-space," Journal of Engineering Physics and Thermophysics, vol. 84, no. 2, pp. 463-478, 2011.

[24] S. Gupta, S. K. Vishwakarma, D. K. Majhi, and S. Kundu, "Possibility of Love wave propagation in a porous layer under the effect of linearly varying directional rigidities," Applied Mathematical Modelling, vol. 37, no. 10-11, pp. 6652-6660, 2013.

[25] P. K. Vaishnav, S. Kundu, S. Gupta, and A. Saha, "Propagation of love-type wave in porous medium over an orthotropic semi-infinite medium with rectangular irregularity," Mathematical Problems in Engineering, vol. 2016, pp. 1-9, 2016.

[26] S. Manna, S. Kundu, and S. Gupta, "Effect of reinforcement and inhomogeneity on the propagation of Love wave," International Journal of Geomechanics, vol. 16, no. 2, Article ID 4015045, 2016.

[27] A. Saha, S. Kundu, S. Gupta, and P. K. Vaishnav, "Effect of irregularity on torsional surface waves in an initially stressed porous layer sandwiched between two non-homogeneous half-spaces," Proceedings of the National Academy of Sciences, India Section A: Physical Sciences, vol. 89, no. 1, pp. 171-183, 2018.

[28] R. Kumhar, S. Kundu, and S. Gupta, "Modelling of Love waves in fluid saturated porous viscoelastic medium resting over an exponentially graded inhomogeneous half-space influenced by gravity," Journal of Applied and Computational Mechanics, vol. 6 , no. 3, pp. 517-530, 2020.

[29] S. Li, G. Zhai, C. Fan, J. Chen, L. Li, and L. Li, "The need for cognition on earthquake risk in China based on psychological distance theory," Complexity, vol. 2020, pp. 1-14, 2020.

[30] J.-y. Ju, Y. Liu, H. Kan, and C. Zhang, "Master-slave composite vibration control of a mobile flexible manipulator via synchronization optimization of observation and feedback," Complexity, vol. 2019, pp. 1-13, 2019.

[31] S. Kundu, S. Gupta, and S. Manna, "Love wave dispersion in pre-stressed homogeneous medium over a porous half-space with irregular boundary surfaces," International Journal of Solids and Structures, vol. 51, no. 21-22, pp. 3689-3697, 2014.

[32] C. D. Wang, H. T. Chou, and D. H. Peng, "Love-wave propagation in an inhomogeneous orthotropic medium obeying the exponential and generalized Power law models," International Journal of Geomechanics, vol. 17, no. 7, Article ID 4017003, 2017.

[33] P. K. Vaishnav, S. Kundu, S. M. Abo-Dahab, and A. Saha, "Love wave behavior in composite fiber-reinforced structure," International Journal of Geomechanics, vol. 17, no. 9, Article ID 6017009, 2017.

[34] S. S. Singh, "Love wave at a layer medium bounded by irregular boundary surfaces," Journal of Vibration and Control, vol. 17, no. 5, pp. 789-795, 2011. 